\title{
Detection of different foreign bodies in the maxillofacial region with spiral computed tomography and cone-beam computed tomography: An in vitro study
}

\author{
Masoud Abolvardi $\mathbb{D}^{1}$, Marzieh Akhlaghian $\mathbb{D}^{2}$, Hadi Hamidi Shishvan $\mathbb{D}^{3, *}$, Farivar Dastan $\mathbb{D}^{4}$ \\ ${ }^{1}$ Department of Oral and Maxillofacial Radiology, Faculty of Dentistry, AJA University of Medical Sciences, Tehran, Iran \\ ${ }^{2}$ Department of Prosthodontics, School of Dentistry, Shiraz University of Medical Sciences, Shiraz, Iran \\ ${ }^{3}$ Department of Oral and Maxillofacial Radiology, Faculty of Dentistry, AJA University of Medical Sciences, Tehran, Iran \\ ${ }^{4}$ Department of Orthodontics, School of Dentistry, Shahed University, Tehran, Iran
}

\section{ABSTRACT}

\begin{abstract}
Purpose: The detection and exact localization of penetrating foreign bodies are crucial for the appropriate management of patients with dentoalveolar trauma. This study compared the efficacy of cone-beam computed tomography (CBCT) and spiral computed tomography (CT) scans for the detection of different foreign bodies composed of 5 frequently encountered materials in 2 sizes. The effect of the location of the foreign bodies on their visibility was also analyzed. Materials and Methods: In this in vitro study, metal, tooth, stone, glass, and plastic particles measuring $1 \times 1 \times 1$ $\mathrm{mm}$ and $2 \times 2 \times 2 \mathrm{~mm}$ were prepared. They were implanted in a sheep's head in the tongue muscle, nasal cavity, and at the interface of the mandibular cortex and soft tissue. CBCT and spiral CT scans were taken and the visibility of foreign bodies was scored by 4 skilled maxillofacial radiologists who were blinded to the location and number of foreign bodies.

Results: CT and CBCT were equally accurate in visualizing metal, stone, and tooth particles of both sizes. However, CBCT was better for detecting glass particles in the periosteum. Although both imaging modalities visualized plastic particles poorly, CT was slightly better for detecting plastic particles, especially the smaller ones.

Conclusion: Considering the lower patient radiation dose and cost, CBCT can be used with almost equal accuracy as $\mathrm{CT}$ for detecting foreign bodies of different compositions and sizes in multiple maxillofacial regions. However, CT performed better for detecting plastic particles.(Imaging Sci Dent 2020; 50: 291-8)
\end{abstract}

KEY WORDS: Cone-Beam Computed Tomography; Tomography, Spiral Computed; Radiography, Dental, Digital; Foreign Bodies

\section{Introduction}

Even the most conservative individuals may experience the penetration of foreign bodies into the soft tissue of the head and neck area, through body cavities or traumatic and iatrogenic injuries; in fact, foreign bodies constitute around $3.8 \%$ of all pathological findings in this region..$^{1-3}$ The composition and location of foreign bodies may vary, depending on the type of trauma. ${ }^{1,4}$ The most frequently detected foreign bodies in the soft tissue of the head and neck are metal, stone, plastic, and glass particles. ${ }^{2,4-6}$ Primary identi-

Received April 1, 2020; Revised August 5, 2020; Accepted August 18, 2020 *Correspondence to : Dr. Hadi Hamidi Shishvan

Department of Oral and Maxillofacial Radiology, Faculty of Dentistry, AJA University of Medical Sciences, Fatemi St., Tehran 1411718541, Iran

Tel) 98-91-4345-1336, E-mail) hadihamidi1336@ gmail.com fication and removal of impacted foreign bodies is critical for preventing complications such as pain, inflammatory reactions, infection, potential injury to the peripheral nerves or vessels, migration to distant areas, impaired wound healing due to granuloma formation, pseudoaneurysms, cellulite and abscess formation, and synovitis. ${ }^{4-9}$ Furthermore, accurate localization is necessary to minimize surgical side effects, especially for foreign bodies that have penetrated deeply or are adjacent to vital structures. ${ }^{3,7,10}$

Several imaging modalities are routinely applied to detect foreign bodies. ${ }^{2,67,11}$ The initial examination includes obtaining a precise history, conducting a clinical examination, and performing conventional radiography, with panoramic radiography generally used as the primary imaging modality for detecting foreign bodies in the head and neck 
region. ${ }^{1,45}$ However, one-third of foreign bodies, particularly non-radiopaque ones, are overlooked on initial examinations, since conventional radiography cannot accurately visualize radiolucent and small radiopaque foreign bodies, especially those in deeper locations, due to the 2-dimensional view and overlapping of shadows with similar densi-

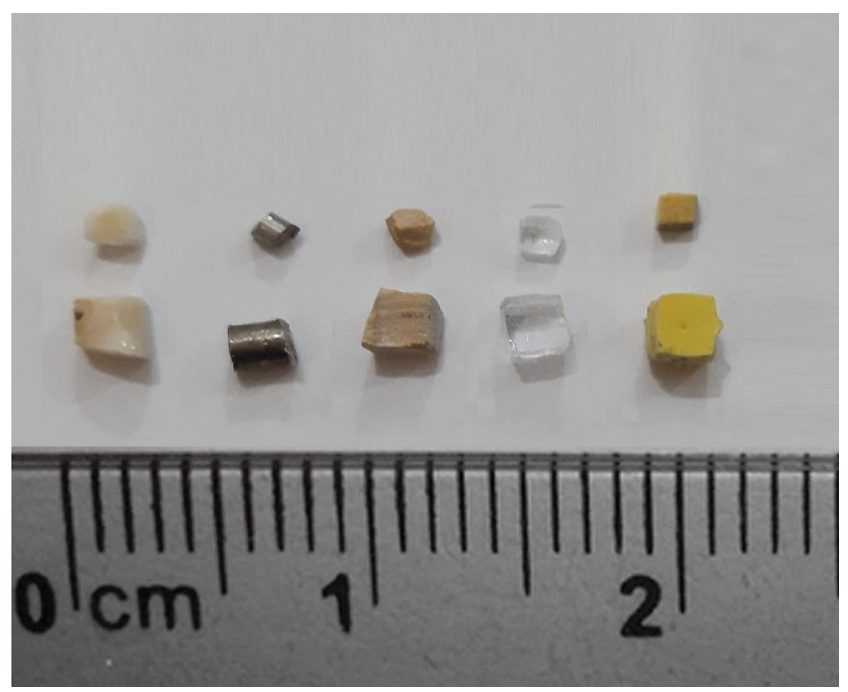

Fig. 1. Different sizes of prepared foreign bodies (tooth, metal, stone, glass, and plastic, from the left to the right). ty. $1,2,6,12$

Ultrasonography is an affordable, easily accessible, real-time imaging technique that works based on ultrasound waves with no radiation exposure. ${ }^{13}$ This modality can effectively display objects in superficial tissues, but not those located adjacent to air, in deep tissues, or behind bony structures. ${ }^{1,2,7,14}$ Magnetic resonance image (MRI) more accurately visualizes radiolucent foreign bodies, but it is initially contraindicated if the type of object is unknown due to the extensive artifacts and the potential danger of further tissue damage caused by displacement of metallic objects within the magnetic field. Moreover, high costs, time consumption, different possible interpretations, and low accuracy restrict the use of MRI in patients who have experienced dentoalveolar trauma. ${ }^{11-13}$

Computed tomography (CT) and cone-beam computed tomography (CBCT) are only indicated when conventional radiography cannot provide enough information for treatment planning and to remove superimposed structures. ${ }^{15}$ In the literature, $\mathrm{CT}$ is considered to be the gold standard for the detection of foreign bodies due to its multi-planar scans, high contrast, accuracy, and proper reconstruction of the shape, size, and position of objects. However, it is quite costly, has limited availability, exposes the patient to
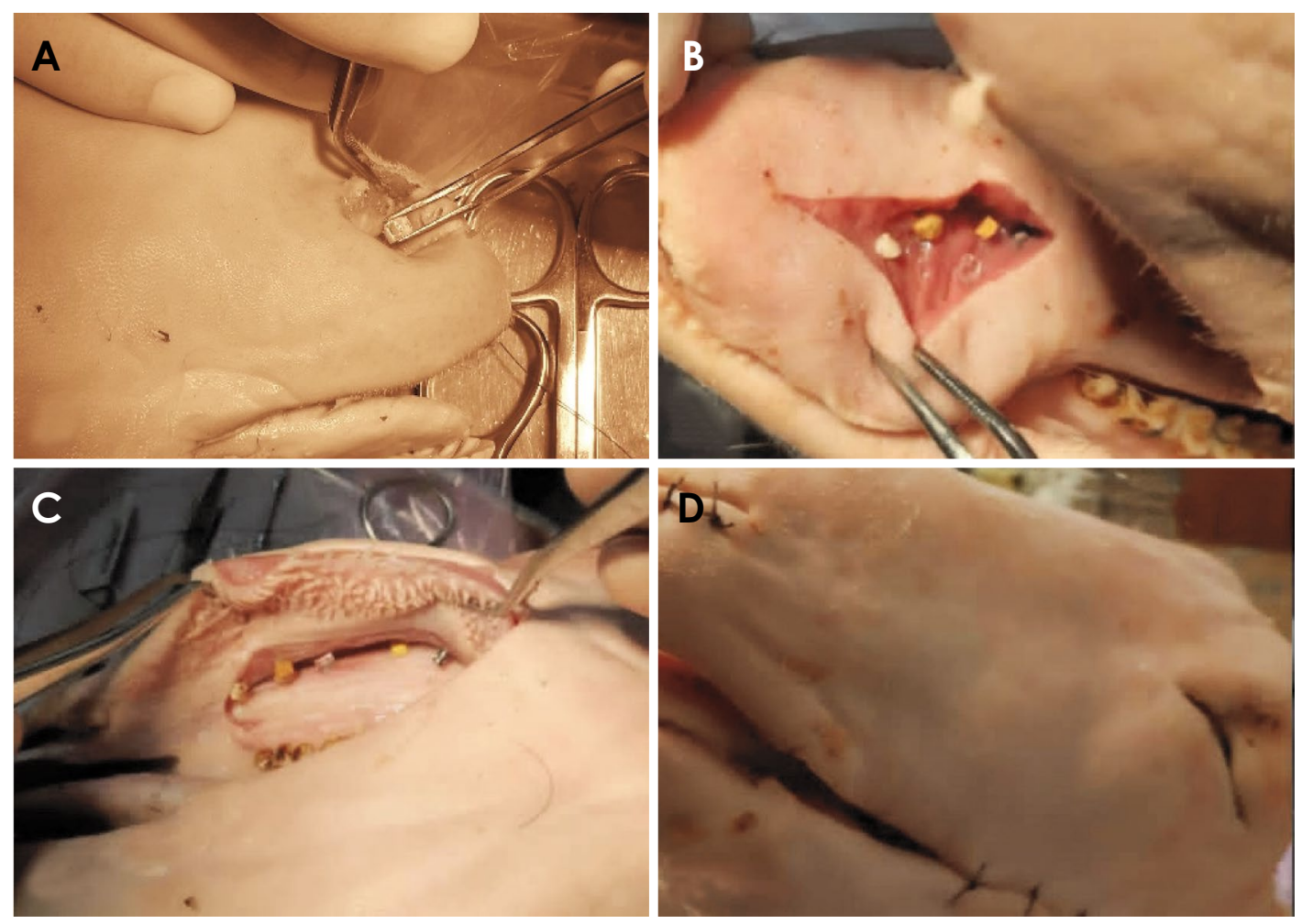

Fig. 2. Foreign bodies are embedded in the nasal cavity. A. Tongue muscle. B. Interface of mandibular cortex and soft tissue. C and D. Sheep's head after the insertion of foreign bodies and suturing. 


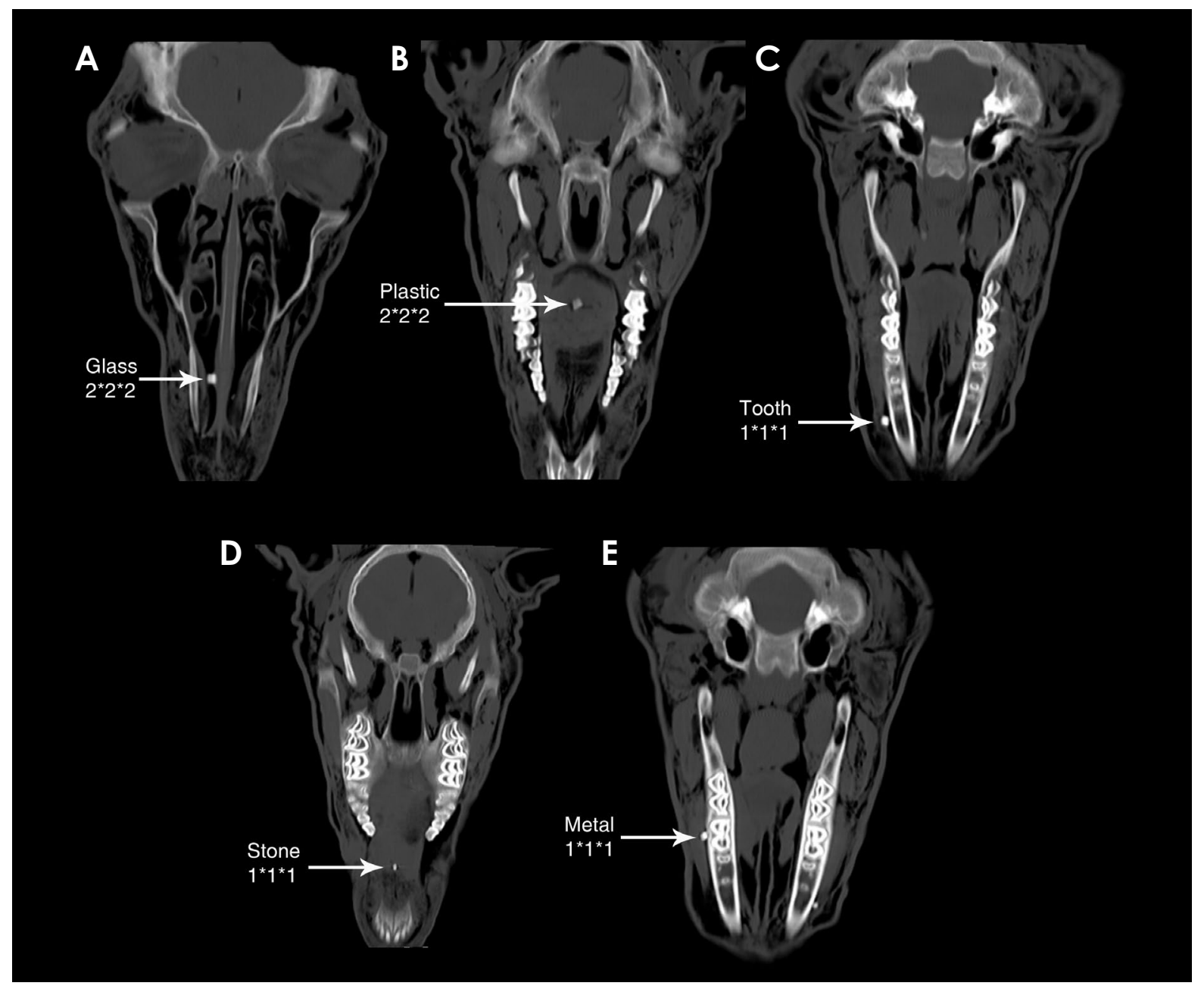

Fig. 3. Detection of foreign bodies on spiral computed tomography. A. Glass particle in the nasal cavity. B. Plastic particle in the tongue. C. Tooth particle in the periosteum. D. Stone particle in the tongue. E. Metal particle in the periosteum.

high doses of ionizing radiation, and is subject to metal artifacts. ${ }^{2,48}$

CBCT, as a novel tomographic method for maxillofacial imaging, has many advantages over conventional CT. It exposes the patient to a lower effective dose of ionizing radiation, ${ }^{15}$ is less costly and time-consuming, is more widely available at dental and maxillofacial radiology centers, and has submillimeter resolution. ${ }^{2,7,16}$ Nonetheless, the drawbacks of CBCT include the geometric projection of the cone beam, the sensitivity of the detectors, and the lower contrast resolution compared to CT. Due to the shortcomings of CBCT for the accurate detection of foreign bodies in soft tissues, the ideal modality remains a matter of debate. $^{11,12}$

This in vitro study was designed to compare the efficacy of CBCT and spiral CT scans in the detection of different foreign bodies of 5 frequently encountered materials in 2 sizes. The effect of the location of foreign bodies (soft tissue, air cavity, or periosteum) on their visibility was also analyzed.

\section{Materials and Methods}

This in vitro study used a fresh sheep's head, 1 day after the animal's death, as a sample for evaluating the materials, considering all applicable institutional and governmental ethical concerns regarding the use of animals under national guidelines and regulations. The sample head was initially scanned to rule out the presence of any possible foreign bodies or anomalies. All the experiments and imaging processes were done on the same day.

As the most commonly detected foreign bodies in the head and neck area, objects of 5 different materials - metal (steel), tooth (human extracted tooth, both enamel and dentin), stone (pebble from a mountain), glass (car windshield), and plastic (condensed rubber from tires) - were 


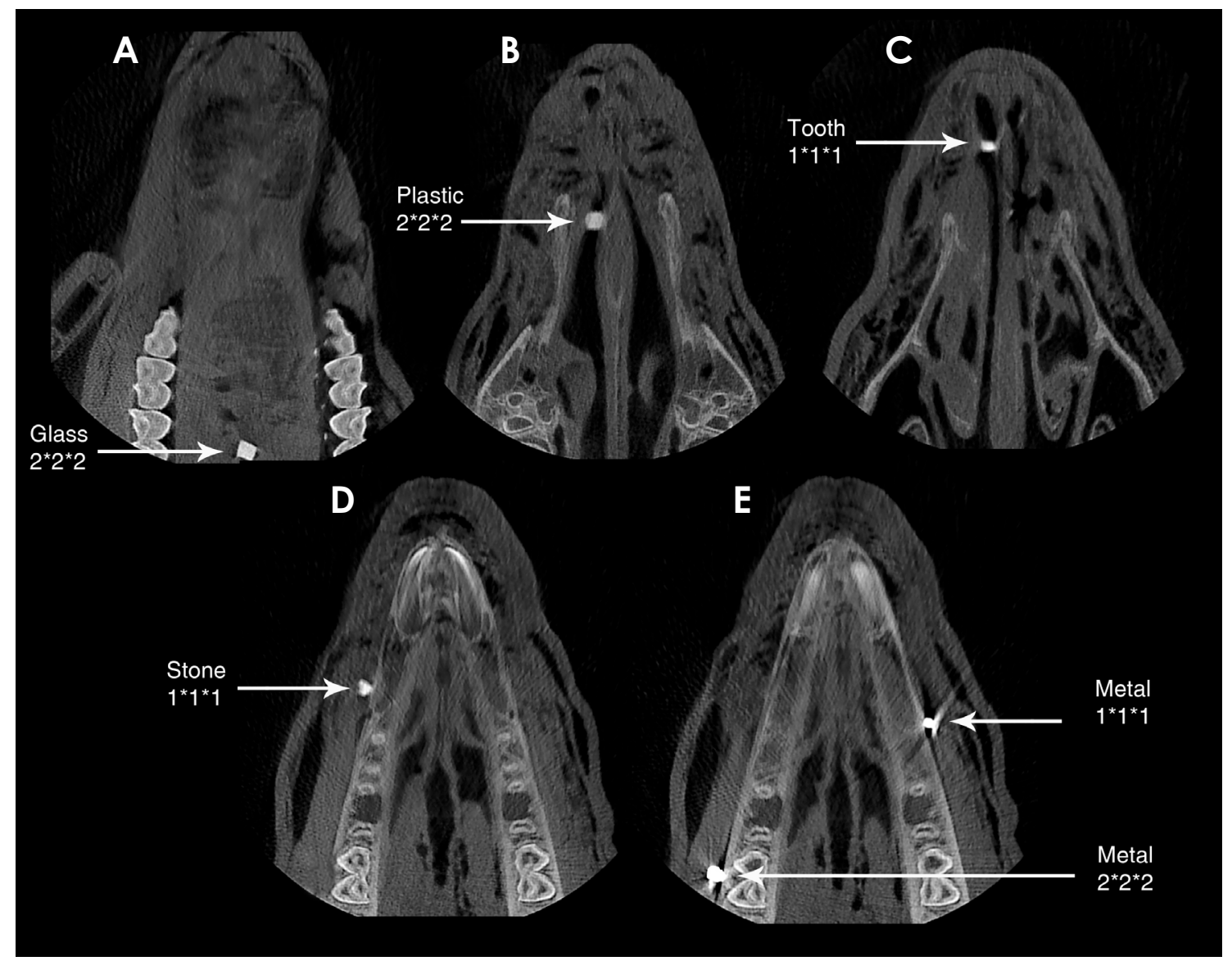

Fig. 4. Detection of foreign bodies on cone-beam computed tomography. A. Glass particle in the nasal cavity. B. Plastic particle in the tongue. C. Tooth particle in the periosteum. D. Stone particle in the tongue. E. Metal particle in the periosteum.

prepared with sizes of $1 \mathrm{~mm} \times 1 \mathrm{~mm} \times 1 \mathrm{~mm}$ and $2 \mathrm{~mm} \times 2$ $\mathrm{mm} \times 2 \mathrm{~mm}$ (Fig. 1). They were scanned with spiral CT to measure their radiopacity and that of the surrounding tissues in terms of Hounsfield units (HU). Then, the objects were separately implanted in the tongue, nasal cavity, and adjacent bone (Fig. 2). Nothing was embedded in the brain and some slots in the 3 mentioned locations were left empty; however, the observers were kept blind to these facts, enabling the assessment of false positivity and the validity of their findings.

To mimic foreign bodies in soft tissue, small incisions were made in the ventral body of the tongue muscle using a \#15 scalpel and objects were gently inserted into the slots and sutured. Objects were also inserted into the nasal cavity to mimic foreign bodies in air by means of a hemostat without any incision, and the nostril skin was sutured. To simulate foreign bodies in the periosteum, a deep incision was made at the vestibular depth of the sheep's mandible to separate the soft tissue from the mandibular cortex; the particles were inserted on the bone and the soft tissue was sutured in its baseline position.

CBCT and spiral CT scans were performed, as these were the most common imaging modalities for head and neck trauma, and axial slices are usually used for further assessment (Figs. 3 and 4).

The spiral CT scans were taken with a Somatom Sensation 16 CT machine (Siemens, Forcheim, Germany), with a matrix size of $512 \times 512$ and $0.4-\mathrm{mm}$ resolution. Scanning was done with the conventional settings for the human head $(110 \mathrm{kVp}, 110 \mathrm{~mA}$, and a minimum slice thickness of $0.6 \mathrm{~mm}$ ). Reconstruction and interpretation were performed with a dedicated software package (Leonardo Work Station Syngo DynaCT software; Siemens Healthineers Headquarters, Erlangen, Germany).

The CBCT images were captured with a NewTom VG machine (NEWTOM VGi, QR srl, Verona, Italy), with a cone X-ray beam, a $1920 \times 1536$-pixel flat panel detector, $15 \mathrm{~cm} \times 15-\mathrm{cm}$ detector size, and $360^{\circ}$ rotation. The slices were prepared at $110 \mathrm{kVp}, 3 \mathrm{~mA}$, a scan time of 18 seconds, and a minimum slice thickness of $1 \mathrm{~mm}$. Recon- 
Table 1. Visibility of different sizes of foreign bodies observed via computed tomography (CT) and cone-beam computed tomography (CBCT) devices in each location

\begin{tabular}{|c|c|c|c|c|c|c|c|}
\hline \multirow{2}{*}{ Material } & \multirow{2}{*}{$\operatorname{Size}(\mathrm{mm})$} & \multicolumn{2}{|c|}{ Nasal cavity (air) } & \multicolumn{2}{|c|}{ Tongue (muscle) } & \multicolumn{2}{|c|}{ Periosteum (adjacent bone) } \\
\hline & & CT & $\mathrm{CBCT}$ & CT & CBCT & CT & CBCT \\
\hline \multirow[t]{2}{*}{ Metal } & $1 \times 1 \times 1$ & Excellent & Excellent & Excellent & Excellent & Excellent & Excellent \\
\hline & $2 \times 2 \times 2$ & Excellent & Excellent & Excellent & Excellent & Excellent & Excellent \\
\hline \multirow[t]{2}{*}{ Tooth } & $1 \times 1 \times 1$ & Excellent & Excellent & Good & Good & Fair & Fair \\
\hline & $2 \times 2 \times 2$ & Excellent & Excellent & Excellent & Excellent & Good & Good \\
\hline \multirow[t]{2}{*}{ Stone } & $1 \times 1 \times 1$ & Excellent & Excellent & Excellent & Excellent & Excellent & Excellent \\
\hline & $2 \times 2 \times 2$ & Excellent & Excellent & Excellent & Excellent & Excellent & Excellent \\
\hline \multirow[t]{2}{*}{ Glass } & $1 \times 1 \times 1$ & Excellent & Excellent & Good & Good & Fair & Good \\
\hline & $2 \times 2 \times 2$ & Excellent & Excellent & Excellent & Excellent & Good & Excellent \\
\hline \multirow[t]{2}{*}{ Plastic } & $1 \times 1 \times 1$ & Fair & Poor & Fair & Poor & Fair & Invisible \\
\hline & $2 \times 2 \times 2$ & Good & Good & Good & Fair & Good & Poor \\
\hline
\end{tabular}

Excellent: excellent visibility, excellent resolution of details, good demarcation from the surroundings. Good: good visibility, good resolution of details, possible demarcation from the surroundings. Fair: fair visibility, insufficient resolution of detail, insufficient demarcation from the surroundings. Poor: poor visibility, details not resolved, poor demarcation from the surroundings. Invisible: invisible, impossible to demarcate from the surroundings

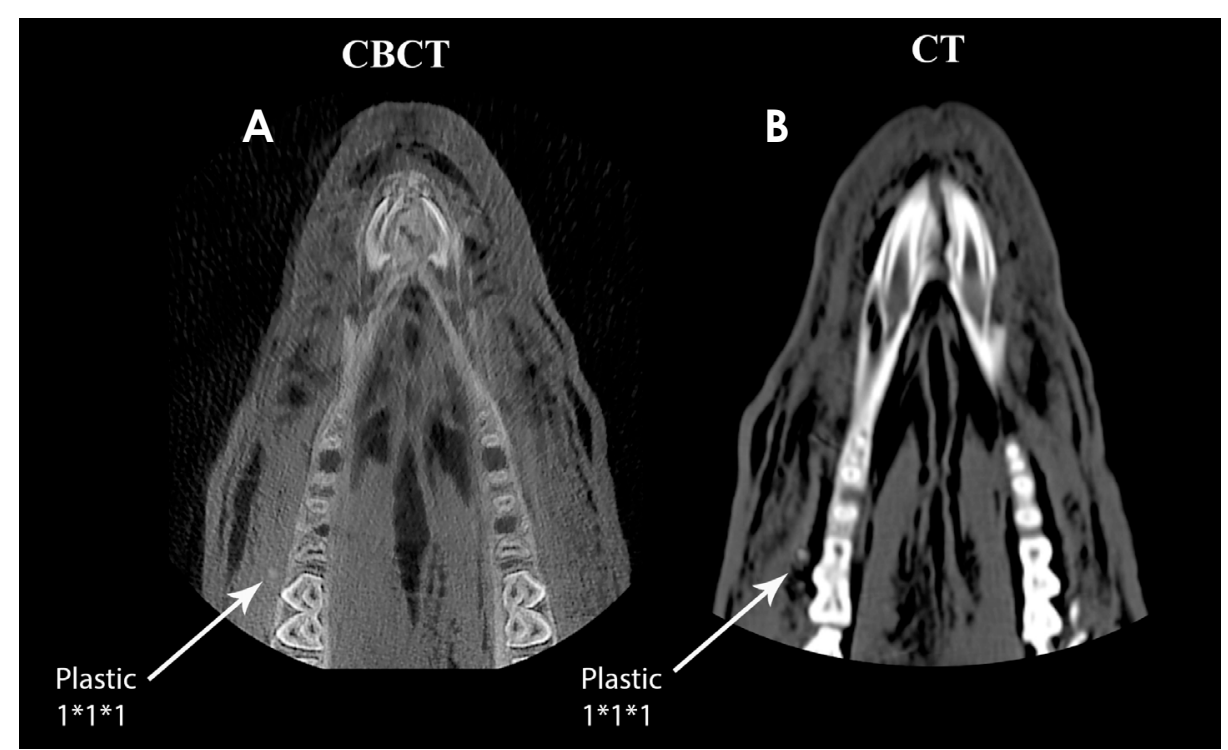

Fig. 5. Difficulty in detecting plastic foreign bodies. Plastic particle in the periosteum on cone-beam computed tomographic (A) and spiral computed tomographic (B) images. struction and assessment were done with the NNT Viewer software, version 2.17 (QR srl, Verona, Italy).

The images were observed by 4 skilled maxillofacial radiologists, who were blinded to the location, presence, and number of foreign bodies. The observers scored the visibility of any possible foreign body at each site using previously described grading criteria. Analyses were repeated at a 2-week interval to determine the reliability of the evaluations. Intraclass correlation coefficients (ICCs) were used to calculate the agreement between the first and second analyses.

\section{Results}

Good agreement was observed between the first and second analyses (ICC $=0.88$ to 0.92 ). The kappa coefficient showed high inter-observer agreement (0.81). The mean values of the results are presented in Table 1. No foreign body was reported in the brain by any of the observers, confirming the validity of the results.

Metal and stone particles of both sizes in all locations were easily detected on both imaging modalities. CBCT and CT yielded the same results in differentiating these materials. The tooth particles of 1 and $2 \mathrm{~mm}$ were well 
detected in the nasal cavity. Those in the tongue were observed with excellent resolution for the 2-mm size and good resolution for the 1-mm size. In the periosteum, $1-\mathrm{mm}$ tooth particles were seen with insufficient resolution, while 2-mm particles were better detected. Overall, $\mathrm{CT}$ and CBCT had equal accuracy in the detection of tooth particles. Glass particles of both sizes were perfectly visualized in the nasal cavity with both imaging modalities. In the tongue and periosteum, 2-mm glass particles were detected better than 1-mm particles. The detection quality in the periosteum was slightly lower than that in other areas. CBCT had better quality than CT in the detection of glass particles in the periosteum. Plastic particles were seen with lower quality than other materials on both imaging modalities. The 2-mm particles were seen with better quality on all images.

Generally, CT was more accurate for detecting plastic particles, especially smaller ones. On CBCT, the 1-mm plastic particles were hardly detected in the nasal cavity and tongue, and could not be differentiated in the periosteum (Fig. 5).

\section{Discussion}

The detection and accurate localization of entrapped foreign bodies are crucial for the appropriate management of dentoalveolar trauma and the prevention of severe consequences. ${ }^{12}$ Whether this is possible depends on the imaging modality that is used, the composition and size of the foreign body, and the location of the foreign body relative to the adjacent structures. ${ }^{2,7,11}$

Several studies have recommended CT as a standard imaging modality for the detection of foreign bodies in the maxillofacial region, as it distinguishes material by measuring HU values, precisely localizes objects, and accurately reconstructs the shape and size of objects, all of which help in the surgical removal of foreign bodies. ${ }^{1,5,11,13,16-20}$ However, CT has pitfalls, including an excessive radiation dose and extensive metal artifacts, which are a particular problem when detecting small metal objects. ${ }^{1,8,2,21} \mathrm{CT}$ has already been replaced by CBCT as the primary method for many 3-dimensional maxillofacial analyses due to its affordability, lower radiation dose, adjustable field of view, fewer metal artifacts, and greater availability in dental practice. $^{4,8,12,21}$

In a comparative study by Abdinian et al., ${ }^{2}$ CBCT was found to be the most accurate technique for detecting foreign bodies, followed by US and panoramic radiography. Likewise, Shokri et al. ${ }^{6}$ concluded that the diagnostic sen- sitivity of CBCT was higher than that of US and MRI. Valizadeh et al. ${ }^{1}$ evaluated CT, CBCT, US, and MRI for visualizing foreign bodies in the maxillofacial area. They recommended US as the first option for locating foreign bodies in the superficial soft tissues, while CBCT (and then, CT) was recommended for locating objects that deeply penetrated the tissues or were located beneath bone. In an in vitro comparison of panoramic radiography, CT, CBCT, MRI, and ultrasonography, Shishvan and Ebrahimnejad $^{11}$ observed that $\mathrm{CT}$ was the most efficient method for detecting different foreign bodies in the maxillofacial area, followed by CBCT. The present in vitro study compared the efficacy of spiral CT and CBCT, which were recommended in several previous studies, to visualize particles of 5 different compositions (all frequently encountered in car accidents) with 2 sizes in 3 common locations. ${ }^{5,20}$

Consistent with previous reports, ${ }^{1,6,7,13,20}$ in the present study, radiopaque foreign bodies, including metal, tooth, stone, and glass particles of both sizes, generated highquality images with both CT and CBCT in both areas. A particle of both enamel and dentin was used to facilitate the differentiation of tooth particles from other expected radiopaque objects in the densely packed structures of the maxillofacial area. ${ }^{13}$

Contrast resolution refers to the distinguishability of anatomical structures with similar contrast, and affects the accuracy of foreign body detection. ${ }^{2}$ The greater scattering of radiation in CBCT causes it to have lower contrast resolution than $\mathrm{CT}^{2}$, which explains the present findings concerning the superiority of $\mathrm{CT}$ for the detection of plastic particles.

CBCT and CT both have higher spatial resolution than other imaging modalities, making them excellent for visualizing tiny objects. ${ }^{2,11}$ Spatial resolution refers to the ability of an imaging system to visualize an object with high contrast, and is limited by pixel and voxel size in CT and CBCT, respectively. ${ }^{7}$ Small foreign bodies are more likely to fill pixels or voxels only partially, making them less detectable. ${ }^{5}$ In the present study, metal and stone particles of both sizes were easily detected, while particles of other materials (especially plastic) were more difficult to detect at a smaller size. Likewise, Abdinian et al. ${ }^{2}$ suggested a direct relationship between particle size and detection accuracy for all imaging modalities. They also reported that CBCT was significantly more accurate than ultrasonography and panoramic radiography for detecting particles of all 3 sizes in their study. The voxel size of CBCT is lower than the pixel size of CT, causing CBCT to have higher spatial resolution. There was no obvious difference between $\mathrm{CT}$ and 
CBCT in the present study, underscoring the ability of both modalities to detect 1-mm and 2-mm particles. Further studies are recommended for the evaluation of smaller foreign bodies.

Foreign bodies that accidentally penetrate into the head and neck region most commonly become lodged in the soft tissue, the interface of bone and soft tissue, or an air-filled cavity. ${ }^{5,20}$ To be vividly visualized, a foreign body should have a radiodensity sufficiently different from that of the surrounding tissues. ${ }^{11}$ Based on the results of the present study, both sizes of metal and stone particles in all of the studied locations were easily observed using both imaging modalities. Tooth particles had the least visibility in the periosteum, probably because of the similar HU values of bone and teeth. ${ }^{13}$ Kaviani et al. ${ }^{7}$ also found that CBCT could detect metal, stone, glass, and tooth particles in the same 3 locations, except for $0.5-\mathrm{mm}$ tooth fragments at the bone surface. For the same reason, in the present study, glass particles in the periosteum were detected with slightly lower quality than those in other areas. CBCT performed better in detecting glass particles in the periosteum. In line with the current findings, Schnider et al. ${ }^{8}$ reported a case of intraoral foreign bodies that were investigated 40 years after a car accident, emphasizing the ability of CBCT to detect and localize glass particles.

Plastic particles were the least visible foreign bodies in the present study, most likely due to the similar radiodensity of plastic and adjacent soft tissues. Similarly, Aras et al. ${ }^{20}$ found that CT could not clearly display non-opaque materials, either between bone and muscle or within muscle. The analysis of objects with low density by Kaviani et al. ${ }^{7}$ also demonstrated lower detectability in the sinus area. Although several studies have shown that radiolucent foreign bodies in superficial tissues were better visualized by ultrasonography than by $\mathrm{CT},{ }^{9,13,20}$ the main challenge when using ultrasonography is the detection of deeply embedded objects. Ultrasonography is not recommended for the evaluation of objects located close to hard tissues, such as bone, or within air-filled cavities, such as the nasal cavity and sinuses. ${ }^{9,13,20}$

The major strengths of the present study are the comparison of CT and CBCT as standard imaging techniques for the accurate detection and localization of foreign bodies, while considering foreign bodies of different compositions, sizes, and locations, as well as including an adequate number of skilled maxillofacial radiologists as raters. However, the in vitro nature of the study prevented reproduction of the inflammatory reactions around the foreign bodies in living tissues. ${ }^{12}$ Furthermore, conducting an in vitro study in a human skull was not ethically allowed in the authors' country; meanwhile, using a dry human skull would have poorly replicated clinical conditions, since a dry skull is an air-filled space with no wet soft tissue. A fresh sheep's head, 1 day after the animal's death, seemed a logical representative of the mammalian body. ${ }^{3}$ Further studies of living humans with foreign bodies are necessary to precisely compare different imaging modalities.

Considering its lower patient radiation dose and cost, $\mathrm{CBCT}$ can be used with almost equal accuracy as CT for the detection of foreign bodies of different sizes and compositions that penetrate to different locations as the result of dentoalveolar trauma. However, plastic particles were better detected using CT. In summary, although CBCT is recommended as the method of choice for detecting foreign bodies in the maxillofacial region, CT is indicated for radiolucent objects.

\section{Acknowledgements}

The authors would like to express their appreciation for Professor Nooroddin Moosavi Nasab for helping with the statistical analyses and to Ms. Farzaneh Rasouli for improving the use of English in this manuscript. Gratitude is also expressed to Dr. Meshksaar Dentomaxillofacial Radiology Center and Department of Radiology of Shahid Rajaee Hospital (Shiraz, Iran) for their cooperation with the imaging processes.

\section{Conflicts of Interest: None}

\section{References}

1. Valizadeh S, Pouraliakbar H, Kiani L, Safi Y, Alibakhshi L. Evaluation of visibility of foreign bodies in the maxillofacial region: comparison of computed tomography, cone beam computed tomography, ultrasound and magnetic resonance imaging. Iran J Radiol 2016; 13: e37265.

2. Abdinian M, Aminian M, Seyyedkhamesi S. Comparison of accuracy between panoramic radiography, cone-beam computed tomography, and ultrasonography in detection of foreign bodies in the maxillofacial region: an in vitro study. J Korean Assoc Oral Maxillofac Surg 2018; 44: 18-24.

3. Lari SS, Shokri A, Hosseinipanah SM, Rostami S, Sabounchi SS. Comparative sensitivity assessment of cone beam computed tomography and digital radiography for detecting foreign bodies. J Contemp Dent Pract 2016; 17: 224-9.

4. Valizadeh S, Alibakhshi L, Ahsaie MG, Kazemi S, Vasegh Z. Diagnostic accuracy of cone beam computed tomography in identification of foreign bodies in the head and neck region. $\mathrm{J}$ Dent Sch Shahid Beheshti Univ Med Sci 2018; 36: 136-9.

5. Eggers G, Mukhamadiev D, Hassfeld S. Detection of foreign 
bodies of the head with digital volume tomography. Dentomaxillofac Radiol 2005; 34: 74-9.

6. Shokri A, Jamalpour M, Jafariyeh B, Poorolajal J, Sabet NK. Comparison of ultrasonography, magnetic resonance imaging and cone beam computed tomography for detection of foreign bodies in maxillofacial region. J Clin Diagn Res 2017; 11: TC15-9.

7. Kaviani F, Javad Rashid R, Shahmoradi Z, Gholamian M. Detection of foreign bodies by spiral computed tomography and cone beam computed tomography in maxillofacial regions. J Dent Res Dent Clin Dent Prospects 2014; 8: 166-71.

8. Schnider N, Reichart PA, Bornstein MM. Intraoral foreign bodies detected 40 years after a car accident using cone beam computed tomography. Quintessence Int 2012; 43: 741-5.

9. Ginsburg MJ, Ellis GL, Flom LL. Detection of soft-tissue foreign bodies by plain radiography, xerography, computed tomography, and ultrasonography. Ann Emerg Med 1990; 19: 701-3.

10. Kadir S, Aronow S, Davis KR. The use of computerized tomography in the detection of intra-orbital foreign bodies. Comput Tomogr 1977; 1: 151-6.

11. Shishvan HH, Ebrahimnejad H. A study on the ability of panoramic, CT, Cone-beam CT, MRI and ultrasonography in detecting different foreign-bodies in the maxillofacial region (an in-vitro study). Electron J Gen Med 2018; 15: em16.

12. Javadrashid R, Golamian M, Shahrzad M, Hajalioghli P, Shahmorady Z, Fouladi DF, et al. Visibility of different intraorbital foreign bodies using plain radiography, computed tomography, magnetic resonance imaging, and cone-beam computed tomography: an in vitro study. Can Assoc Radiol J 2017; 68: 194-201.

13. Javadrashid R, Fouladi DF, Golamian M, Hajalioghli P, Daghighi $\mathrm{MH}$, Shahmorady $\mathrm{Z}$, et al. Visibility of different foreign bodies in the maxillofacial region using plain radiography, CT,
MRI and ultrasonography: an in vitro study. Dentomaxillofac Radiol 2015; 44: 20140229.

14. Gomaa M, Abdelaal A. Ultrasonography versus radiography in detection of different foreign bodies in a cadaveric calf thigh specimen. Res J Vet Pract 2015; 3: 83-8.

15. Kullman L, Al Sane M. Guidelines for dental radiography immediately after a dento-alveolar trauma, a systematic literature review. Dent Traumatol 2012; 28: 193-9.

16. Javadrashid R, Kaviany F, Shahmorady Z, Niknamy M, Golamian M, Sadrarhami S, et al. Evaluation of the result of diagnostic of Spiral computed tomography comparing with cone beam computed tomography in diagnostic of foreign body in the orbit. J Am Sci 2013; 9(7S): 94-8.

17. Krimmel M, Cornelius CP, Stojadinovic S, Hoffmann J, Reinert S. Wooden foreign bodies in facial injury: a radiological pitfall. Int J Oral Maxillofac Surg 2001; 30: 445-7.

18. Ober CP, Jones JC, Larson MM, Lanz OI, Werre SR. Comparison of ultrasound, computed tomography, and magnetic resonance imaging in detection of acute wooden foreign bodies in the canine manus. Vet Radiol Ultrasound 2008; 49: 411-8.

19. Popescu R, Dobrovăţ B, Nemţoi A, Lăduncă O, Haba D. The importance of CT imaging for detecting traumatic intraorbitar and maxillofacial foreign bodies. Rom Neurosurg 2011; 18 : 476-82.

20. Aras MH, Miloglu O, Barutcugil C, Kantarci M, Ozcan E, Harorli A. Comparison of the sensitivity for detecting foreign bodies among conventional plain radiography, computed tomography and ultrasonography. Dentomaxillofac Radiol 2010; 39: 72-8.

21. Eggers G, Mühling J, Hofele C. Clinical use of navigation based on cone-beam computer tomography in maxillofacial surgery. Br J Oral Maxillofac Surg 2009; 47: 450-4. 